\title{
On $\delta$-Primary Fuzzy Hyperideals of Commutative Semihyperrings
}

\author{
Ashraf Abumghaiseeb \\ Department of Mathematics, Islamic University of Gaza, P.O. Box 108, Gaza, Palestine.
}

How to cite this paper: Ashraf Abumghaiseeb. (2021) On $\delta$-Primary Fuzzy Hyperideals of Commutative Semihyperrings. Journal of Applied Mathematics and Computation, 5(4), 315-320.

DOI: 10.26855/jamc.2021.12.009

Received: October 14, 2021

Accepted: November 10, 2021

Published: December 3, 2021

*Corresponding author: Ashraf Abumghaiseeb, Department of Mathematics, Islamic University of Gaza, P.O. Box 108, Gaza, Palestine.

Email: ashrafgaza@gmail.com

\begin{abstract}
In this paper, I give an introduction about the algebraic hyperstructure theory mainly. I study a semihyperring and a hyperideal of a semihyperring, then I study the definition of fuzzy subset of a set $X$ as a function from $X$ to the interval $[0,1]$, and explain the definition of a fuzzy hyperideal of a semihyperring $R$ and primary fuzzy hyperideal. I discuss the $\delta$ function of the fuzzy hyperideal of commutative semihyperring. This function assign to each fuzzy hyperideal $\mu$ of the semihyperring $R$ to another fuzzy hyperideal $\delta(\mu)$ of $R$ such that $\mu \leq \delta(\mu)$ and we defined the $\delta$-primary hyperideal expansion, also we discussed some properties of this expansion. I show that $\delta$-primary fuzzy hyperideal expansion in an intersection preserving. Then I give a definition of fuzzy homomorphism function of two commutative semihyperrings. Finally, I define a global expansion $\delta$ of fuzzy hyperideal and investigate the relation between $\mu$ and $f(\mu)$ under the global expansion $\delta$.
\end{abstract}

\section{Keywords}

Semihyperring, fuzzy hyperideal, $\delta$-primary, fuzzy homomorphism

\section{Introduction}

The first one who introduced the theory of algebraic hyperstructure as a generalization of ordinary algebraic structure was the French mathematician, F. Marty in 1934. Firstly, Marty introduced the concepts of a hypergroup [1]. Marty defined a hyperoperation $\circ$ as a relation from a nonempty set $H$ to $P^{*}(H)$ which is the set of the nonempty subsets of $H$. It can be written as:

$$
\circ: H \times H \rightarrow P^{*}(H) \text {. }
$$

By the definition of a hyperoperation, Marty defined a hypergroup as follows:

If $H$ is a nonempty set and $\circ$ is a hyperoperation defined on $H$, such that $\circ$ satisfies the following conditions,

1. $(x \circ y) \circ z=x \circ(y \circ z)$,

2. $x \circ H=H \circ x$,

for all $x, y, z \in H$, then $(H, \circ)$ is a hypergroup where,

$$
x \circ(y \circ z)=\bigcup_{r \in(y \circ z)} x \circ r, x \circ H=\bigcup_{h \in H} x \circ h .
$$

In 1956, Marc Krasner introduced the concepts of the hyperrings and hyperfields and he generalized the additive structure of the hyperring as "canonical hypergroup" [2]. Since then, many researchers have studied the theory of hyperstructure and many results have been published on hyperrings, most of these results where extensions of the standard results in commutative algebra.

In 2003, B. Davvaz extended the isomorphism theorems [3], and in 2006, [4] Davvaz and Salasi extended the Chinese 
Remainder The orem to the Hyperrings. In 2011, Asokkumar and Velrajan proved isomorphism theorems for hyperrings with two hyperoperations [5]. These papers introduced revelation to look for other important results from commutative algebra that can be transferred to hyperrings.

The fuzzy set theory is a branch of mathematics. In 1965, Lotfi Zadeh defined the fuzzy subset of any set $X$ as a function $\mu: X \rightarrow[0,1][6]$. After that, the students of Zadeh introduced the basic concepts of fuzzy algebra. In this paper, we define the expansion of fuzzy hyperideal and generalize the expansion theory on hyperideals of semihyperring to fuzzy hyperideals of semihyperring.

\section{Preliminaries}

Definition 2.1 [7] A semihyperring is an algebraic structure $(R,+, \cdot)$ which satisfies the following axioms:

1) $(R,+)$ is commutative hypermonoid, i.e.

(i) $(x+y)+z=x+(y+z)$ for all $x, y, z \in R$,

(ii) There exists $0 \in R$, such that $x+0=0+x=x$ for all $x \in R$,

(iii) $x+y=y+x$ for all $x, y \in R$.

2) $(R, \cdot)$ is a semigroup, i.e.

(i) $0 \cdot x=x \cdot 0=0$ for all $x \in R$.

(ii) $(x \cdot y) \cdot z=x \cdot(y \cdot z)$ for all $x, y, z \in R$.

3) The multiplication is distributive to the hyperaddition, so that

$x \cdot(y+z)=x \cdot y+x \cdot z,(x+y) \cdot z=x \cdot y+x \cdot z$ for all $x, y, z \in R$.

Definition 2.2 [7] A semihyperring $(R,+, \cdot)$ is called:

(i) Commutative, if $x \cdot y=y \cdot x$ for all $x, y \in R$.

(ii) Semihyperring with identity if $1_{R} \in R$, such that $x \cdot 1_{R}=1_{R} \cdot x=x$, for all $x \in R$.

Definition 2.3 [7] A left (right) hyperideal of a semihyperring $\mathrm{R}$ is a nonempty subset $\mathrm{I}$ of $\mathrm{R}$, such that:

(i) For all $\mathrm{x}, \mathrm{y} \in \mathrm{I}, \mathrm{x}+\mathrm{y} \subseteq \mathrm{I}$,

(ii) For all $x \in I, r \in R, r \cdot x \in I(x \cdot r \in I)$, a hyperideal of a semihyperring is a non-empty subset of $R$, which is left and right hyperideal of $\mathrm{R}$, and the set of all hyperideals of $\mathrm{R}$ is denoted by $\operatorname{Id}(\mathrm{R})$.

Definition 2.4 [7] For $x \in R$, if there exist one and only one $-x \in R$ such that $0 \in x+(-x)$ then $-x$ is called the opposite of $x$. Denote the set of all opposite elements of $R$, by $V(R)$, that is $V(R)=\{x \in R \mid$ there exists $y \in$ $R, 0 \in x+y\}$.

Definition 2.5 A semihyperring $R$ is called an additively reversive if it satisfy the reversive property with respect to the hyperoperation of addition [7], i.e. for $a \in b+c$ implies $b \in a+(-c)$ and $c=a+(-b)$.

Definition 2.6 Let $R$ be a commutative semihyperring and $I$ be a hyperideal of $R$. Then the closure of $I$ which is denoted by $c l(I)$, is defined by $c l(I)=\{a \in R: a+c \subseteq I$, for some $c \in I\}$.

\section{3. $\delta$-Primary Fuzzy Hyperideal of Commutative Semihyperring}

Definition 3.1 [3] Let $X$ be a set, a fuzzy subset $\mu$ of $X$ is a function from $X$ to [0,1], such that $0 \leq \mu(x) \leq 1$ for all $x \in X$. For any two fuzzy sets $\mu, \gamma$ we say that $\mu \leq \gamma$ if and only if $\mu(x) \leq \gamma(x)$ for all $x \in X,(\mu \cap \gamma)(x)=$ $(\mu \wedge \gamma)(x)=\min \{\mu(x), \gamma(x)\}$, and $(\mu \cup \gamma)(x)=(\mu \vee \gamma)(x)=\max \{\mu(x), \gamma(x)\}$.

Example 3.1 Let $\mathbb{R}$ be the set of real numbers and let $\mu: \mathbb{R} \rightarrow[0,1]$ defined by

$$
\mu(x)=\left\{\begin{array}{lr}
1 & \text { if } x=0 \\
1-\frac{1}{x} & \text { if } x>0 \\
1+\frac{1}{x} & \text { if } x<0
\end{array}\right.
$$

Then, $\mu$ is a fuzzy subset of real numbers $\mathbb{R}$.

Definition 3.2 [9] Let $R$ be a semihyperring and let $\mu$ be a fuzzy set of $R$, then $\mu$ is said to be a fuzzy hyperideal of $R$ if:

i) $\quad \mu(x y) \geq \mu(x) \vee \mu(y)$ for all $x, y \in R$.

ii) $\quad \underset{z \in x+y}{\inf } \mu(z) \geq \mu(x) \wedge \mu(y)$ for all $x, y \in R$.

Definition 3.3 Let $R$ be a semihyperring and let $\mu, \gamma$ be fuzzy hyperideals of $R$, let $\delta$ be a function that assigns each fuzzy hyperideal $\mu$ another fuzzy hyperideal $\delta(\mu)$ of $R$ such that:

i) $\quad \mu \leq \delta(\mu)$. 
ii) if $\mu \leq \gamma$ then $\delta(\mu) \leq \delta(\gamma)$.

then $\delta$ is called a fuzzy hyperideal expansion.

Definition 3.4 [10]: Let $\mu$ be a fuzzy hyperideal of a semihyperring $R, \mu$ is called primary if for any $a, b \in R$, either $\mu(a b)=\mu(a)$ or $\mu\left(b^{n}\right) \geq \mu(a b)$ for some $n \in \mathbb{N}$.

Definition 3.5: Let $\delta$ be a fuzzy hyperideal expansion, a fuzzy hyperideal $\mu$ is called $\delta$-primary if $\mu(a b)>\mu(a)$ then $\delta(\mu(b)) \geq \mu(a b)$. Also, the definition can be written as, if $\mu(a b)>\delta(\mu(a))$ then $\mu(b) \geq \mu(a b)$.

Definition 3.6 [8] Let $\mu$ be a fuzzy set of a semihyperring $R, t \in[0,1]$, then $\mu_{t}=\{x \in R: \mu(x) \geq t\}$ is called the level set of $\mu$.

Definition 3.7 [11] Let $\mu$ be a fuzzy hyperideal of a semihyperring $R$, then $\rho(\mu)$, called the radical of $\mu$ is defined by $\rho(\mu(x))=\sup _{n \geq 1} \mu\left(x^{n}\right), n \in \mathbb{N}$.

Theorem 3.1: If $\mu$ is a fuzzy hyperideal of a semihyperring $R$, then $\rho(\mu)$ is a fuzzy hyperideal of $R$.

Proof. Since $\mu$ is a fuzzy hyperideal, then $\mu\left(x^{n} y^{n}\right) \geq \mu\left(x^{n}\right) \vee \mu\left(y^{n}\right)$, so that $\rho(\mu(x y))={ }_{n \geq 1}^{\text {sup }} \mu\left(x^{n} y^{n}\right) \geq{ }_{n \geq 1}^{\text {sup }} \mu\left(x^{n}\right) \vee{ }_{n \geq 1}^{\text {sup }} \mu\left(x^{n}\right)=\rho(\mu(x)) \vee \rho(\mu(y))$. Consider that $\mu(x+y)=\{\mu(z): z \in x+y\}$, so that

$$
\begin{aligned}
& \inf _{z \in x+y} \rho(\mu(z))=\inf _{z \in x+y}\left({ }_{n \geq 1}^{\sup } \mu\left(z^{n}\right)\right)=\inf \left({ }_{n \geq 1}^{\sup } \mu(x+y)^{n}\right)
\end{aligned}
$$

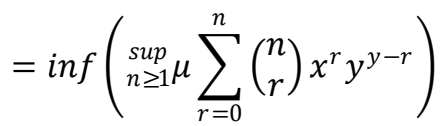

$$
\begin{aligned}
& \geq\left({ }_{n \geq 1}^{\sup }\left(\min _{1 \leq r \leq n} \mu\left(x^{r} y^{n-r}\right)\right)\right) \\
& \geq\left({ }_{n \geq 1}^{\sup } \mu\left(x^{r_{n}} y^{n-r_{n}}\right)\right), \quad 0 \leq r_{n} \leq n,
\end{aligned}
$$

where $\mu\left(x^{r_{n}} y^{n-r_{n}}\right)=\min _{\substack{1 \leq r \leq n \\ \text { inf }}} \mu\left(x^{r} y^{n-r}\right)$. If $r_{n}$ bounded above i.e $r_{n} \leq k$ for some constant $k$, and $n-r_{n}$ is unbounded above then $\inf _{z \in x+y} \rho(\mu(z)) \geq \sup _{n \geq 1} \mu\left(x^{r_{n}} y^{n-r_{n}}\right) \geq{ }_{n \geq 1}^{\sup } \mu\left(y^{n-r_{n}}\right)$, which is true for all values of $n$,

$\operatorname{so}_{z \in x+y} \rho(\mu(z)) \geq{ }_{n \geq 1}^{\sup } \mu\left(y^{n}\right)=\rho(\mu(y))$. Similarly, if $n-r_{n}$ is bounded above and $r_{n}$ is unbounded, then

$\underset{z \in x+y}{\inf } \rho(\mu(z)) \geq{ }_{n \geq 1}^{\sup } \mu\left(x^{n}\right)=\rho(\mu(x))$, if none of $n-r_{n}, r_{n}$ is bounded then $\inf _{z \in x+y} \rho(\mu(z)) \geq \rho(\mu(x)) \wedge \rho(\mu(y))$, hence $\rho(\mu)$ is a fuzzy hyperideal of $R$.

Example 3.2

1) For any fuzzy hyperideal $\mu$ of a semihyperring $R, \delta_{0}(\mu)=\mu$ is a fuzzy hyperideal expansion.

2) $\delta_{1}(\mu)=\rho(\mu)$ is a fuzzy hyperideal expansion.

Proof. 1. It is trivial case.

2. For all $x \in R$ we have $\delta(\mu(x))=\rho(\mu(x))={ }_{n \geq 1}^{\sup } \mu\left(x^{n}\right) \geq \mu\left(x^{n-1} x\right) \geq \mu(x)$, also if $\mu_{1}, \mu_{2}$ are fuzzy hyperideals such that $\mu_{1} \leq \mu_{2}$, then $\delta\left(\mu_{1}(x)\right)=\rho\left(\mu_{1}(x)\right)={ }_{n \geq 1}^{\sup } \mu_{1}\left(x^{n}\right) \leq{ }_{n \geq 1}^{\sup } \mu_{2}\left(x^{n}\right)=\rho\left(\mu_{2}\right)=\delta\left(\mu_{2}(x)\right)$. Hence $\delta(\mu)=$ $\rho((\mu))$ is a fuzzy hyperideal expansion.

Definition 3.8 [10] Let $\mu$ be a fuzzy hyperideal of a semihyperring $R$, then, $\mu$ is called prime if for all $x, y \in R$ we have $\mu(x y)=\mu(x)$ or $\mu(x y)=\mu(y)$.

Theorem 3.2 Let $\mu$ be a fuzzy hyperideal of a semihyperring $R$, then $\mu$ is prime if and only if $\mu$ is $\delta_{0}$-primary.

Proof. Assume that $\mu$ is a prime fuzzy hyperideal, let $\mu(a b)>\mu(a)$ so that $\mu(a b)=\mu(b)$, then $\delta_{0}(\mu(b))=$ $\mu(b) \geq \mu(a b)$ and $\mu$ is $\delta_{0}$-primary. Conversely, let $\mu$ is $\delta_{0}$-primary, and $\mu(a b)>\mu(a)$, then $\delta_{0}(\mu(b))=\mu(b) \geq$ $\mu(a b)$, but $\mu(a b) \geq \mu(b)$. So we have $\mu(a b)=\mu(b)$ and $\mu$ is a fuzzy prime hyperideal.

Theorem 3.3: Let $\mu$ be a fuzzy hyperideal of a semihyperring $R$, then $\mu$ is primary if and only if $\mu$ is $\delta_{1}$-primary.

Proof. Assume that $\mu$ be a primary fuzzy hyperideal with $\mu(a b) \neq \mu(a)$, then $\mu(a b)>\mu(a)$. So that there exists $n \in \mathbb{N}$ such that $\mu\left(a^{n}\right) \geq \mu(a b)$, then $\delta(\mu(a))={ }_{n \geq 1}^{\sup } \mu\left(a^{n}\right)$. Therefore $\mu$ is $\delta_{1}$-primary. Conversely, suppose that $\mu$ is $\delta_{1}$-primary with $\mu(a b) \neq \mu(a)$, then $\mu(a b)>\mu(a)$, so that $\rho(\mu(b))=\sup _{n \geq 1} \mu\left(b^{n}\right) \geq \mu(a b)$. If $\mu\left(b^{n}\right)<\mu(a b)$ for all $n \in \mathbb{N}$, then $\sup _{n \geq 1} \mu\left(b^{n}\right)<\mu(a b)$ which is a contradiction, so that there exists $n \in \mathbb{N}$, such that $\mu\left(b^{n}\right) \geq \mu(a b)$ and so $\mu$ is a primary fuzzy hyperideal.

Theorem 3.4 Let $\mu$ be a fuzzy hyperideal of a semihyperring $R$, and $\delta$ be a fuzzy hyperideal expansion and $\gamma$ be a hyperideal expansion such that $\gamma\left(\mu_{t}\right)=(\delta \mu)_{t}, t \in[0,1]$. Then $\mu$ is a $\delta$-primary fuzzy hyperideal if and only if $\mu_{t}$ is a $\gamma$-primary hyperideal of a semihyperring $R$.

Proof. Suppose that $\mu$ is a $\delta$-primary fuzzy hyperideal of $R$. Let $x y \in \mu_{t}$ with $y \notin \mu_{t}$, then $\mu(x y) \geq t$ but $\mu(y)<t$. So that $\mu(x y)>\mu(y)$, then $\delta \mu(y) \geq \mu(x y) \geq t$. Therefore, $y \in(\delta \mu)_{t}=\gamma\left(\mu_{t}\right)$. Hence, $\mu_{t}$ is a $\gamma$-primary 
hyperideal of $R$.

Conversely, assume that $\mu_{t}$ is a $\gamma$-primary hyperideal of $R$. Let $\mu(x y)>\mu(y)$, then $\mu(x y)=t$ for some $t \in[0,1]$. Then $y \notin \mu_{t}$, but $x y \in \mu_{t}$ so that $y \in \gamma\left(\mu_{t}\right)=(\delta \mu)_{t}$. We get $\delta \mu(y) \geq t=\mu(x y)$. Therefore, $\mu$ is a $\delta$-primary fuzzy hyperideal.

Theorem 3.5 Let $\delta, \gamma$ be fuzzy hyperideals expansions of a semihyperring $R$ such that $\delta(\mu) \subseteq \gamma(\mu)$ for every fuzzy hyperideal $\mu$. If $\mu$ is $\delta$-prrimary, then $\mu$ is $\gamma$-primary. Also every prime fuzzy hyperideal $\mu$ is a $\delta$-primary fuzzy hyperideal.

Proof. Suppose that $\mu$ is a $\delta$-primary fuzzy hyperideal, such that $\mu(a b)>\mu(b)$, then $\delta \mu(b) \geq \mu(a b)$. So that $\gamma \mu(b) \geq \delta \mu(b) \geq \mu(a b)$. Therefore, $\mu$ is $\gamma$-primary. Now, assume that $\mu$ is a prime fuzzy hyperideal with $\mu(a b)>$ $\mu(b)$, so that $\mu(a b)=\mu(a)$, but we know that $\delta \mu \geq \mu$, so that $\delta \mu(a) \geq \mu(a)=\mu(a b)$. Hence, $\mu$ is a $\delta$-primary fuzzy hyperideal.

Theorem 3.6 The intersection of two fuzzy hyperideal expansions is also a fuzzy hyperideal expansion.

Proof. Assume that $\delta_{1}, \delta_{2}$ are two fuzzy hyperideal expansions of the semihyperring $R$. Let $\delta$ be defined as $\delta=\delta_{1} \cap \delta_{2}$. Then for any fuzzy hyperideal $\mu$ we have that $\mu \subseteq \delta_{1} \mu, \mu \subseteq \delta_{2} \mu$, so that $\mu \subseteq \delta_{1} \mu \cap \delta_{2} \mu=\delta(\mu)$. Now suppose $\sigma$ is another fuzzy hyperideal of $R$ such that $\mu \subseteq \sigma$. Then $\delta \mu \subseteq \delta_{1} \mu \subseteq \delta_{1} \sigma$ and $\delta \mu \subseteq \delta_{2} \mu \subseteq \delta_{2} \sigma$, which implies that $\delta \mu \subseteq \delta_{1} \sigma \cap \delta_{2} \sigma=\delta \sigma$. Hence $\delta$ is a fuzzy hyperideal expansion.

Remark. The previous theorem can be generalized for finite fuzzy hyperideal expansions that are the intersection of a finite number of fuzzy hyperideal expansions is also a fuzzy hyperideal expansion.

Theorem 3.7 Let $\delta$ be a fuzzy hyperideal expansion of a semihyperring $R$, and $E_{\delta}$ be defined by $E_{\delta}(\mu)=\cap\{\rho: \rho$ is $\delta$-primary a fuzzy hyperideal expansion, $\mu \subseteq \rho$ \}. Then, $E_{\delta}$ is a fuzzy hyperideal expansion.

Proof. By the definition of $E_{\delta}$ we have that $\mu \subseteq E_{\delta}(\mu)$. Now suppose that $\mu_{1} \subseteq \mu_{2}$, then any $\delta$-primary fuzzy hyperideal $\rho$ that contains $\mu_{2}$ is also contains $\mu_{1}$. Since $\mu_{1} \subseteq \mu_{2}$, then $E_{\delta}\left(\mu_{1}\right) \subseteq E_{\delta}\left(\mu_{2}\right)$. So that $E_{\delta}$ is a fuzzy hyperideal expansion.

Definition 3.9 [12] Let $\delta$ be a fuzzy hyperideal expansion, then $\delta$ is called intersection preserving if $\delta(\mu \cap \rho)=$ $\delta(\mu) \cap \delta(\rho)$ for all fuzzy hyperideals $\mu, \rho$ of a semihyperring $R$.

Example $3.3 \delta_{0}$ is intersection preserving because $\delta_{0}(\mu \cap \rho)=\mu \cap \rho=\delta_{0}(\mu) \cap \delta_{0}(\rho)$. Also, $\delta_{1}$ is intersection preserving because $\delta_{1}(\mu \cap \rho)=\rho(\mu \cap \rho)={ }_{n \geq 1}^{\sup }(\mu \wedge \rho)\left(x^{n}\right)={ }_{n \geq 1}^{\sup }(\mu)\left(x^{n}\right) \wedge_{n \geq 1}^{\sup _{n}}(\rho)\left(x^{n}\right)=\delta_{1}(\mu) \cap \delta_{1}(\rho)$.

Theorem 3.8 Let $\delta$ be an intersection preserving fuzzy hyperideal expansion and $\mu_{1}, \mu_{2}, \cdots, \mu_{n}$ be $\delta$-primary fuzzy hyperideals such that $\delta\left(\mu_{i}\right)=\delta\left(\mu_{j}\right)=\rho$ for $1 \leq i, j \leq n$. Let

$$
\mu=\bigcap_{i=1}^{n} \mu_{i} .
$$

Then $\mu$ is a $\delta$-primary fuzzy hyperideal.

Proof. Suppose that $\mu(a b)>\mu(b)$, then $\mu(a b)=\Lambda_{i=1}^{n} \mu_{i}(a b)>\Lambda_{i=1}^{n} \mu_{i}(a)$. So that $\mu(a b)>\mu_{j}(a)$ for some $j$. So we have $\mu_{j}(a b)>\Lambda_{i=1}^{n} \mu_{i}(a)=\mu(a) \geq \mu_{j}(a)$ and since $\mu_{j}$ is $\delta$-primary. Then $\delta \mu_{j}(b) \geq \mu(a b)$. Since $\delta$ is intersection preserving, then

$$
\delta(\mu)=\delta\left(\bigcap_{i=1}^{n} \mu_{i}\right)=\left(\bigcap_{i=1}^{n} \delta \mu_{i}\right)=\bigcap_{i=1}^{n} \rho=\rho .
$$

Therefore, $\mu(b)=\rho(b)=\delta \mu_{j}(b) \geq \mu(a b)$. Hence $\mu$ is a $\delta$-primary fuzzy hyperideal.

Definition 3.10 [13] Let $R, S$ be nonempty subsets, and $\theta \in[0,1), f$ be a fuzzy subset of the Cartesian cross product $R \times S$. Then $f$ is called a fuzzy function if:

1) For all $x \in R$ there exists $y \in S$, such that $f(x, y)>\theta$.

2) For all $x \in R$, for all $y_{1}, y_{2} \in S, f\left(x, y_{1}\right)>\theta$ and $f\left(x, y_{2}\right)>\theta$ implies that $y_{1}=y_{2}$.

Definition 3.11 [13] Let $R, S$ be nonempty subsets, $\theta \in[0,1)$ and $f$ be a fuzzy function defined on $R \times S$. Then;

1) $f$ is onto if for all $y \in S$ there exists $x \in R$ such that $f(x, y)>\theta$.

2) $f$ is one-to-one if for all $x_{1}, x_{2} \in R$, for all $y \in S, f\left(x_{1}, y\right)>\theta, f\left(x_{2}, y\right)>\theta$ implies that $x_{1}=x_{2}$.

Definition 3.12 Let $R, S$ be commutative semihyperrings, and $f$ be a fuzzy function defined on $R \times S$. Then $f$ is said to be a fuzzy homomorphism if and only if for all $x_{1}, x_{2} \in R$ and for all $y \in S$

1) $\underset{z \in x_{1}+x_{2}}{\inf } f(z, y) \geq \sup \left(\operatorname{minf}\left\{f\left(x_{1}, y_{1}\right), f\left(x_{2}, y_{2}\right)\right\} \mid y \in y_{1}+y_{2}, y_{1}, y_{2} \in S\right\}$

2) $f\left(x_{1} x_{2}, y\right) \geq \sup \left\{\inf \left\{f\left(x_{1}, y_{1}\right), f\left(x_{2}, y_{2}\right)\right\} \mid y=y_{1} y_{2}, y_{1}, y_{2} \in S\right\}$.

The homomorphism $f$ is said to be a fuzzy isomorphism if it is one-to-one and onto. 
Definition 3.13 [13] Let $f$ be a fuzzy subset of $\times S$, then the inverse of $f$ is $f^{-1}$ on $S \times R$ is defined by $f^{-1}(s, r)=f(r, s)$ for all $(s, r) \in S \times R$.

Definition 3.14 [13] Let $R, S$ be semihyperrings and $f$ be a fuzzy function of $R$ into $S$.

1) If $\mu$ is a fuzzy subset of $R$, then the fuzzy subset $f(\mu)$ of $S$ defined by for all $s \in S, f(\mu)(s)=$ $\sup \{q(r): f(r, s)>\theta\}$.

2) If $\lambda$ is a fuzzy subset of $S$, then the fuzzy subset $f^{-1}(\lambda)$ of $R$ defined by for all $r \in R, f^{-1}(\lambda)(r)=$ $\lambda(s)$ where $f(r, s)>\theta$.

Theorem 3.9 Let $R, S$ be semihyperrings, and $f$ be a fuzzy homomorphism from $R$ onto $S$. Then;

1) If $\mu$ is a fuzzy hyperideal of $R$ then $f(\mu)$ is a fuzzy hyperideal of $S$.

2) If $\lambda$ is a fuzzy hyperideal of $S$ then $f^{-1}(\lambda)$ is a fuzzy hyperideal of $R$.

Proof. 1) Assume that $\mu$ is a fuzzy hyperideal of $R$. Since $f$ is onto homomorphism, then for each $x, y \in S$ there exists $r_{1}, r_{2} \in R$ such that $f\left(r_{1}, x\right)>\theta, f\left(r_{2}, y\right)>\theta$. So that

$$
\begin{gathered}
f(\mu)(x y)=\sup \{\mu(r): f(r, x y)>\theta\} \geq \sup \left\{\mu\left(r_{1} r_{2}\right): f\left(r_{1} r_{2}, x y\right)>\theta\right\} \\
\geq \sup \left\{\mu\left(r_{1}\right) \vee \mu\left(r_{2}\right): f\left(r_{1} r_{2}, x y\right)>\theta\right\} \\
\geq \sup \left\{\mu\left(r_{1}\right): f\left(r_{1}, x\right)>\theta\right\} \vee \sup \left\{\mu\left(r_{2}\right): f\left(r_{2}, y\right)>\theta\right\} \\
=f(\mu)(x) \vee f(\mu)(y) .
\end{gathered}
$$

Also, $\inf _{s \in x+y} f(\mu)(s)=\inf \left(\sup _{s \in x+y}\left\{\mu\left(r_{1} r_{2}\right): f\left(r_{1} r_{2}, s\right)>\theta\right\}\right)$

$$
\begin{gathered}
\geq \inf \left(\sup \left\{\mu\left(r_{1}\right): f\left(r_{1}, x\right)>\theta\right\} \wedge \sup \left\{\mu\left(r_{2}\right): f\left(r_{2}, x\right)>\theta\right\}\right) \\
=\sup \left\{\mu\left(r_{1}\right): f\left(r_{1}, x\right)>\theta\right\} \wedge \sup \left\{\mu\left(r_{2}\right): f\left(r_{2}, x\right)>\theta\right\} \\
=f(\mu)(x) \wedge f(\mu)(y) .
\end{gathered}
$$

Therefore, $f(\mu)$ is a fuzzy hyperideal of $R$.

2) Suppose that $\lambda$ is a fuzzy hyperideal of $S$, then for $x, y \in R$ we have,

$$
\begin{aligned}
& f^{-1}(\lambda)(x y)=\lambda\left(s_{1} s_{2}\right) \text { where } f\left(x y, s_{1} s_{2}\right)>\theta \\
& \geq\left\{\lambda\left(s_{1}\right) \vee \lambda\left(s_{2}\right): f\left(x y, s_{1} s_{2}\right) \geq \sup \left\{f\left(x, s_{1}\right) \wedge f\left(y, s_{2}\right)\right\}\right. \\
& \geq\left\{\lambda\left(s_{1}\right): f\left(x, s_{1}\right)>\theta\right\} \vee\left\{\lambda\left(s_{2}\right): f\left(y, s_{2}\right)>\theta\right\} \\
& =f^{-1}(\lambda)(x) \vee f^{-1}(\lambda)(y) \text {. } \\
& \inf _{z \in x+y} f^{-1}(\lambda)(z)=\inf \left\{\lambda\left(s_{1} s_{2}\right): f\left(z, s_{1} s_{2}\right)>\theta\right\} \\
& \geq \inf \left\{\lambda\left(s_{1}\right) \vee \lambda\left(s_{2}\right): f(z, s) \geq f\left(x, s_{1}\right) \vee f\left(y, s_{2}\right), s \in s_{1}+s_{2}\right\} \\
& \geq \inf \left\{\left\{\lambda\left(s_{1}\right): f\left(x, s_{1}\right)>\theta\right\} \vee\left\{\lambda\left(s_{2}\right): f\left(y, s_{2}\right)>\theta\right\}\right. \\
& =\lambda\left(s_{1}\right) \wedge \lambda\left(s_{2}\right)=f^{-1}(\lambda)(x) \wedge f^{-1}(\lambda)(y) .
\end{aligned}
$$

Hence, $f^{-1}(\lambda)$ is a fuzzy hyperideal of $R$.

Definition 3.15 Let $f$ be a fuzzy semihyperring homomorphism from $R$ into $S$, and $\delta$ be a fuzzy hyperideal expansion. Then, $\delta$ is said to be a global expansion if $\delta\left(f^{-1}(\lambda)\right)=f^{-1}(\delta(\lambda))$ for any fuzzy hyperideal $\lambda$ of $S$.

Theorem 3.10 Let $R, S$ be semihyperrings, and $f$ be a fuzzy homomorphism from $R$ into $S$ and $\delta$ be a global fuzzy hyperideal expansion. If $\mu$ is a $\delta$-primary fuzzy hyperideal of $S$, then $f^{-1}(\mu)$ is a $\delta$-primary fuzzy hyperideal of $R$.

Proof. By Theorem 3.9, we have that $f^{-1}(\mu)$ is a fuzzy hyperideal of $R$ whenever $\mu$ is a fuzzy hyperideal of $S$. Assume that $f^{-1}(\mu)(a b)>f^{-1}(\mu)(a)$, then $\mu(x y)>\mu(x)$ where $f(a b, x y)>\theta$ and $f(a, x)>\theta$, but $\mu$ is $\delta$-primary, so that $\delta(\mu(y)) \geq \mu(x y)$ which implies that $f^{-1}(\delta(\mu(b))) \geq f^{-1}(\mu(a b))$ and since $\delta$ is global, we have $\delta\left(f^{-1}(\mu(b))\right) \geq f^{-1}(\mu(a b))$. Therefore $f^{-1}(\mu)$ is a $\delta$-primary fuzzy hyperideal of $R$.

Definition 3.14 [13] Let $f$ be a fuzzy semihyperring homomorphism from $R$ to $S, \theta \in[0,1)$, then the kernel of $f$ is $\operatorname{Ker}(f)=\{x \in R: f(x, 0)>\theta\}$.

Theorem 3.11 Let $f$ be a fuzzy semihyperring homomorphism from $R$ onto $S$ and $\delta$ be a global fuzzy hyperideal expansion. The fuzzy hyperideal $\mu$ of $R$ is $\delta$-primary then $f(\mu)$ is a $\delta$-primary fuzzy hyperideal of $S$.

Proof. By Theorem $3.9 \mu$ is a fuzzy hyperideal of $R$ then $f(\mu)$ is a fuzzy hyperideal of $S$. Now, suppose that $f(\mu)(s r)=\mu(a b), f(a b, s r)>\theta$, since $f$ is homomorphism, then $f(\mu)(s)=\mu(a)$ and $f(\mu)(r)=\mu(b)$. Assume 
that $\mu$ is $\delta$-primary and $f(\mu)(s r)>f(\mu)(s)$. Then $\mu(a b)>\mu(a)$, so $\delta(\mu(b)) \geq \mu(a b)$ and $f(\delta(\mu(r))) \geq f(\mu(s r))$, and since $\delta$ is global. Then, $\delta(f(\mu(r))) \geq f(\mu(s r))$. So $f(\mu)$ is $\delta$-primary fuzzy hyperideal of $S$.

Theorem 3.12 Let $f$ be a fuzzy semihyperring homomorphism from $R$ onto $S$ and $\delta$ be a global fuzzy hyperideal expansion. Then $f$ induces one to one corresponding between $\delta$-primary fuzzy hyperideals of $R$ and $\delta$-primary fuzzy hyperideals of $S$.

Proof. The proof is a direct result of Theorem 3.10 and Theorem 3.11.

\section{References}

[1] Omidi, S. and Davvaz, B. (2017). “Ordered Krasner Hyperrings”. Iranian Journal of Mathematical Sciences and Informatics. Vol. 12, No. 2, pp. 35-49.

[2] Shojaei H. and Fasino D. (2019). Isomorphism Theorems in the Primary Categories of Krasner Hypermodules. https://www.mdpi.com/journal/symmetry.

[3] Davvaz, B. and Salasi, A. (2006). "A realization of hyperrings.” Communications in Algebra, 34(12): 4389-4400.

[4] Ostadhadi-Dehkordi, S. and Davvaz, B. (2016). "A note on isomorphism theorems of Krasner (m, n)-hyperrings”. Arab. J. Math., 5: 103-115.

[5] Asokkumar, A. (2013). "Derivations in Hyperrings and Prime Hyperrings". Iranian Journal of Mathematical Sciences and Informatics, 8(1): 1-13.

[6] Zadeh, L. A. (1965). "Fuzzy set Information and Control”. 8(1): 338-358.

[7] Shabir, M., Mehmood, N., and Corsini, P. (2010). "Semihyperrings Characterized by Their Hyperideals". Italian Journal of Pure and Applied Mathematics.

[8] Kazanci, O., YJlmaz, S., and Davvaz, B. (2019). "On Fuzzy Ordered Hyperideals in Ordered Semihyperrings". https://www.hindawi.com/journals/afs/2019/3693926/.

[9] Ahmed, A. and Aslam, M. (2013). “On Fuzzy Semihyperring”. https://arxiv.org/pdf/1304.6371, 22 Apr, 2013.

[10] Kumar, R. (1992). “Certain fuzzy ideals of rings redefined”. Fuzzy Sets and Systems, 46(2): 251-260.

[11] Mukherjee, T. K. and Sen, M. K. (1993). "Primary fuzzy ideals and radical of fuzzy ideals”. Fuzzy Sets and Systems, 56(1), 97-101.

[12] Dongsheng, Z. (2001). “ $\delta$-primary ideals of commutative rings”. Kyunkpook Math. Journal, 41(1): 17-22.

[13] Malik, D. S. and Mordeson, J. N. (1992). Fuzzy homomorphisms of rings. Fuzzy Sets and Systems, 46(1): 139-146. 